\title{
Energy expenditure, body composition and dietary habits in progressive supranuclear palsy
}

\author{
Marina Picillo ${ }^{1}\left[\right.$ - Maria Francesca Tepedino ${ }^{1} \cdot$ Maria Claudia Russillo ${ }^{1} \cdot$ Filomena Abate $^{1} \cdot$ Marta Savastano $^{1}$. \\ Antonio De Simone ${ }^{1} \cdot$ Roberto Erro $^{1} \cdot$ Maria Teresa Pellecchia ${ }^{1} \cdot$ Paolo Barone ${ }^{1}$
}

Received: 30 July 2021 / Revised: 21 September 2021 / Accepted: 11 October 2021 / Published online: 21 October 2021

(c) The Author(s), under exclusive licence to Springer-Verlag GmbH Germany 2021

\begin{abstract}
Introduction Little is known about metabolic changes in progressive supranuclear palsy. Goals of the present study are to: (1) investigate whether early progressive supranuclear palsy is associated with changes in energy expenditure, body composition and dietary intake compared with Parkinson's disease and healthy controls; (2) assess the accuracy of the Harris-Benedict equation to predict measured rest energy expenditure in progressive supranuclear palsy; (3) verify differences according to sex, phenotypes, disease severity and presence of dysphagia in progressive supranuclear palsy.

Methods Twenty-one progressive supranuclear palsy, 41 Parkinson's disease and nine healthy controls were included. Rest energy expenditure was assessed with indirect calorimeter, body composition with bio-impedance analysis and physical activity and dietary intake were estimated with a validated frequency questionnaire. Parametric testing was used to analyze differences between groups.

Results Progressive supranuclear palsy showed reduced total daily energy expenditure and physical activity compared to both other cohorts $(p<0.001)$ and a tendency toward lower fat-free mass compared to Parkinson's disease $(p>0.05)$. Limited accuracy was shown for the Harris-Benedict equation (accurate prediction frequency $<60 \%$ ). Greater disease severity was associated with lower rest energy expenditure $(p=0.030)$, fat-free mass $(p=0.026)$ and muscle mass $(p=0.029)$.

Conclusion Greater disease severity is associated with reduction in rest energy expenditure likely due to the reduction in lean mass and muscle mass. Such data may pave the way to clinical trials evaluating the efficacy of muscle-targeted nutritional support and physical therapy in preserving muscle mass and improving motor performances in progressive supranuclear palsy at early stages.
\end{abstract}

Keywords Progressive supranuclear palsy $\cdot$ Rest energy expenditure $\cdot$ Body composition $\cdot$ Diet $\cdot$ Parkinson

\section{Introduction}

Recent evidence suggests that neurodegenerative diseases are associated with a wide spectrum of metabolic changes [1-6]. Furthermore, alterations in eating behaviors and dietary habits often feature patients with Parkinson's disease (PD) and behavioral variant of Frontotemporal dementia (bvFTD) since the earliest stages [3, 4].

Marina Picillo mpicillo@unisa.it

1 Center for Neurodegenerative Diseases (CEMAND), Department of Medicine, Surgery and Dentistry "Scuola Medica Salernitana", University of Salerno, Via Allende, 84081 Baronissi, Salerno, Italy
While PD and Alzheimer's disease have been associated with weight loss, bvFTD has been linked to weight gain [4]. However, the nature of these metabolic changes and how they can affect the disease progression are largely unknown [4].

Scant of data is available on progressive supranuclear palsy (PSP), a rapidly progressive 4-R tauopathy classified within the spectrum of Frontotemporal lobar degeneration (FTLD) with bvFTD and Amyotrophic Lateral Sclerosis (ALS). Studies on limited number of PSP patients report a tendency toward weight loss since the earliest phase of the disease and a decreased muscle energy metabolism [7, 8].

Aim of the present case-control study was to investigate whether PSP at early stages is associated with changes in energy expenditure, body composition and dietary intake compared with PD patients and healthy controls (HC) 
matched for age, sex and body mass index (BMI) (and disease duration for PD). Additional objectives were to (1) assess the accuracy of the equation of Harris-Benedict, constructed to estimate rest energy expenditure in healthy individuals, to predict measured in PSP; (2) verify differences in the above-mentioned variables according to pre-specified categories in the PSP cohort (sex, phenotypes, disease severity and presence of clinically significant dysphagia).

\section{Methods}

\section{Participants}

Consecutive patients with PSP diagnosed with the Movement Disorder Society (MDS) criteria and referred to the Center for Neurodegenerative diseases (CEMAND) of the University of Salerno, Italy, were approached to take part to the present study between June 2018 and April 2021 [9]. Detailed information on enrollment and application of the PSP diagnostic criteria and phenotyping is available elsewhere [10-13]. Specific inclusion criteria for the present study were: ability to walk with for at least 10 steps with or without unilateral support, ability to complete the proposed assessments based on current physical and cognitive conditions, availability of a caregiver supporting the compilation of the dietary habit questionnaire.

A cohort of patients with idiopathic PD, diagnosed according to the MDS criteria [14], was recruited matched by gender, age, disease duration and BMI (PSP:PD $=1: 2$ ) (Flow chart showing enrollment in Supplemental File 1).

Finally, a cohort of HC matched for age, sex and BMI was recruited among spouses and caregivers of patients attending the outpatient clinic (PSP:HC $=1: 0.5$ ).

Based on previous literature [3], the following exclusion criteria were considered for all enrolled cohorts: inclusion in any intervention trial, history of significant weight loss $(>5 \mathrm{~kg}$ ) in the previous 3 months, uncontrolled diabetes, major respiratory diseases, hepatic and renal failure, thyroid diseases, and all other conditions/diseases that can affect energy expenditure, body composition or dietary intake (e.g., fever, infections, cancer). In addition, PSP and PD patients with changes in dopaminergic medications during the previous three months as well as PD patients with motor fluctuations and dyskinesia were excluded.

\section{Assessments}

\section{Anthropometry}

Body weight (to the nearest $0.5 \mathrm{~kg}$ ) was measured using the same calibrated flat scale (SECA GMBH 7997021099;
Germany) according to standard procedure. Height was measured in $\mathrm{cm}$ (to the nearest $0.5 \mathrm{~cm}$ ) and BMI was derived as the ratio between weight $[\mathrm{kg}]$ and height $[\mathrm{m}]$ squared $\left(\mathrm{kg} / \mathrm{m}^{2}\right)$ [15]. Measurement of waist circumference was performed with an elastic tape measure at the midpoint between the last rib and the iliac crest [15].

\section{Energy expenditure}

Rest energy expenditure was measured, based on oxygen consumption (VO2), with the portable FitMate $\AA$ (Cosmed, Rome, Italy) calorimeter and estimated according to the validated and widely used gender-specific predictive equation of Harris-Benedict for healthy people based on weight, height and age. Details on rest energy expenditure evaluation as well as computation of the total daily energy expenditure and physical activity level are given in Supplemental file 1 .

\section{Body composition}

Bio-impedance analysis (BIA Akern 101) was used to measure body composition. The following parameters were retrieved from the output of the BIA instrument: Fat-Free Mass (Kg), Fat-Free Mass index (kg/m), Fat Mass (Kg), Fat Mass index $(\mathrm{Kg} / \mathrm{m})$, Body Cellular Mass $(\mathrm{Kg})$, Body Cellular Mass index $(\mathrm{Kg} / \mathrm{m})$, Total Body Water $(\mathrm{L})$, Total Body Water index (L/m), Extracellular Water (L). Furthermore, whole-body Skeletal Muscle Mass was estimated from impedance parameters using the equation provided by Janssen et al. [16]. Then, Skeletal Muscle Mass was normalized for height (muscle mass $[\mathrm{kg}] /$ height $[\mathrm{m}]^{2}$ ) and skeletal muscle mass index (Skeletal Muscle Mass/height $[\mathrm{m}]^{2}$ ) was calculated to define low muscle mass as follows: men $<8.87 \mathrm{~kg} /$ $\mathrm{m}^{2}$ and women $<6.42 \mathrm{~kg} / \mathrm{m}^{2}$ [17].

\section{Dietary habits}

Dietary habits were estimated with specific questions from a validated online software freeware (Supplemental File 1).

\section{Disease-specific features}

Severity of disease was assessed with the Movement Disorder Society-sponsored version of the Unified Parkinson's disease part III (MDS-UPDRS-III) [18] in both PSP and PD and with the PSP rating scale (PSP-rs) in PSP [19]. PSP patients were divided in two groups according to the median PSP-rs total score (less severe disease $<36$; more severe disease $\geq 36$ ).

Only for PSP, severity of dysphagia was derived from the item 13 of the PSP-rs and a score equal or greater than 2 was considered a proxy for clinical significant dysphagia [19]. None of the PD patients reported clinically significant 
dysphagia (no cough or choking episodes or need to modify the food consistency).

Dopaminergic therapy was reported as Levodopa Equivalent Daily Dose (LEDD).

\section{Statistical analysis}

Kolmogorov-Smirnov tests were run to determine suitability of variables for parametric analyses. Between-groups comparisons were performed with the Student's $t$ test, ANOVA, ANCOVA or with the Fisher's exact test, as appropriate. Post hoc analyses were performed with the Bonferroni test. As for the PSP cohort, pre-specified sub-group analyses by sex, phenotypes, disease severity (PSP-rs $<36$ versus $\geq 36$ ) and clinically significant dysphagia (PSP-rs item $13<2$ versus $\geq 2$ ) were also considered.

The agreement between measured and estimated rest energy expenditure in PSP was investigated through calculation of bias and the limits of agreement by the method of Bland and Altman, and the Lin's concordance correlation coefficient $[20,21]$. The accuracy of predictive equations was also described as the proportion of patients outside the threshold of accurate prediction of $\pm 10 \%$ [21].

All analyses were performed using SPSS 27.0 (SPSS Inc., Chicago, Illinois). All statistical tests were two-tailed, and a $\mathrm{p}<0.05$ was deemed as statistically significant.

\section{Results}

Upon exclusion of 13 patients (7 PSP for physical and cognitive conditions preventing completion of study assessments, 3 PSP for lack of caregiver, 1 PSP for uncontrolled diabetes, 1 PSP for thyroid problems and 1 PD for uncontrolled diabetes) and $1 \mathrm{HC}$ for incomplete data, $21 \mathrm{PSP}, 41 \mathrm{PD}$ and 9 $\mathrm{HC}$ were included in the present study. Table 1 shows demographic, clinical and anthropometric features of the study cohorts. Two out of 21 (9.5\%) PSP and 11/41 (26.8\%) PD were not taking any dopaminergic medication. As expected, given the greater disease severity, PSP patients presented higher LEDD and MDS-UPDRS-III compared to PD.

Seventeen PSP patients (80.9\%) presented a Richardson's syndrome (PSP-RS), two PSP with predominant parkinsonism and two PSP with progressive gait freezing. Twelve PSP patients $(57.1 \%)$ reported clinically significant dysphagia.

Table 2 details comparisons between groups for energy expenditure, body composition and dietary intake.

\section{Energy expenditure and accuracy of Harris- Benedict equation}

No significant differences in either measured or estimated rest energy expenditure were observed between cohorts. However, total daily energy expenditure was significantly lower in PSP compared to both PD and HC, due to lower physical activity level and physical activity intensity levels $(p<0.001)$. Of note, the difference between PSP and PD remained significant after correction for LEDD $(p<0.001)$. Such results were confirmed when comparing PSP men with
Table 1 Demographic, clinical and anthropometric features of the study cohorts

\begin{tabular}{lllll}
\hline & PSP $(21)$ & PD (41) & HC (9) & $p$ \\
\hline Age, years & $67.05(6.31)$ & $63.54(10.58)$ & $62.22(6.47)$ & 0.270 \\
Sex, men, $N(\%)$ & $12(57.1)$ & $30(73.1)$ & $5(55.5)$ & 0.347 \\
$\begin{array}{l}\text { Disease duration from onset of } \\
\quad 3.14(2)\end{array}$ & $2.49(2.16)$ & - & 0.255 \\
symptoms & $501.84(255.84)$ & $265.12(326.86)$ & - & \\
LEDD, mg & $39.29(15.93)$ & - & - & $\mathbf{0 . 0 0 7}$ \\
MSP-rs & $35.9(15.11)$ & $26.34(8.89)$ & - & - \\
Weight, Kg & $72.12(12.66)$ & $79.8(14.22)$ & $84(27.95)$ & 0.109 \\
BMI, kg/m ${ }^{2}$ & $27.91(4.76)$ & $28.48(4.22)$ & $27.84(5.46)$ & 0.865 \\
BMI categories & & & & 0.860 \\
$\quad$ Normal weight, $n(\%)$ & $5(26.3)$ & $8(21.6)$ & $3(33.3)$ & \\
Overweight, $n(\%)$ & $10(52.6)$ & $19(51.4)$ & $3(33.3)$ & \\
Obese, $n(\%)$ & $4(21.1)$ & $10(27)$ & $3(33.3)$ & \\
Waist circumference, cm & $97.57(11.24)$ & $101.78(17.6)$ & $93.78(8.75)$ & 0.284 \\
\hline
\end{tabular}

Significant differences are highlighted in bold

All data are expressed in mean (standard deviation), unless otherwise specified

$B M I$ body mass index; $H C$ healthy controls; $L E D D$ levodopa equivalente daily dose; MDS-UPDRS-III the Movement Disorder Society version of the Unified Parkinson's disease part III; PD Parkinson's disease; PSP Progressive supranuclear palsy (PSP); PSP-rs Progressive supranuclear palsy rating scale 
Table 2 Study assessments in PSP, PD and HC

\begin{tabular}{|c|c|c|c|c|}
\hline & PSP (21) & $\mathrm{PD}(41)$ & $\mathrm{HC}(9)$ & $p$ \\
\hline \multicolumn{5}{|l|}{ Energy Expenditure } \\
\hline Measured rest energy expenditure, Kcal/day & $1696.43(425.59)$ & $1702.76(439.54)$ & $1635.56(494.05)$ & 0.917 \\
\hline Estimated rest energy expenditure, Kcal/day & $1439.96(317.74)$ & $1562.5(264.12)$ & $1430.99(225.18)$ & 0.177 \\
\hline Total daily energy expenditure, Kcal/day & $2721.96(603.23)$ & $3614.87(932.22)$ & $3652.6(674.96)$ & $<0.001 *$ \\
\hline Physical activity level & $1.61(0.17)$ & $2.17(0.48)$ & $2.38(0.67)$ & $<\mathbf{0 . 0 0 1}^{\S}$ \\
\hline Physical activity intensity levels, $n(\%)$ & & & & $<0.001^{\circ}$ \\
\hline $1.40-1.69$ low & $13(68.4)$ & $6(15.4)$ & $2(22.2)$ & \\
\hline $1.70-1.99$ moderate & $5(26.3)$ & $10(25.6)$ & $1(11.1)$ & \\
\hline $2.00-2.40$ high & $1(5.3)$ & $23(59)$ & $6(66.7)$ & \\
\hline \multicolumn{5}{|l|}{ Body composition } \\
\hline Free Fat Mass, $\mathrm{Kg}$ & $54.31(9.11)$ & $59.49(10.06)$ & $51.11(8.18)$ & $\mathbf{0 . 0 2 6}^{+}$ \\
\hline Free Fat Mass index, $\mathrm{Kg} / \mathrm{m}$ & $33.59(4.15)$ & $35.54(4.69)$ & $31.43(3.76)$ & $\mathbf{0 . 0 3 1}^{+}$ \\
\hline Skeletal Muscle Mass Index, $\mathrm{Kg} / \mathrm{m}^{2}$ & $9.76(1.55)$ & $10.34(1.69)$ & $8.91(1.52)$ & 0.053 \\
\hline Pathologic Skeletal Muscle Mass Index, $n(\%)$ & $2(9.5)$ & $2(5)$ & $2(22.2)$ & 0.245 \\
\hline Fat Mass, $\mathrm{Kg}$ & $17.77(9.64)$ & $19.58(8.91)$ & $21.73(9.86)$ & 0.544 \\
\hline Fat Mass index, $\mathrm{Kg} / \mathrm{m}$ & $11.17(6.32)$ & $11.81(5.53)$ & $13.48(6.45)$ & 0.618 \\
\hline Body Cellular Mass, Kg & $27.41(7)$ & $31.3(7.38)$ & $25.98(4.73)$ & $\mathbf{0 . 0 3 9}^{+}$ \\
\hline Body Cellular Mass index, $\mathrm{Kg} / \mathrm{m}$ & $16.93(3.89)$ & $18.67(3.8)$ & $15.95(2)$ & 0.064 \\
\hline Total Body Water, L & $40.12(6.93)$ & $44.45(7.68)$ & $37.76(6.48)$ & $\mathbf{0 . 0 1 7}^{\wedge}$ \\
\hline Total body Water index, $\mathrm{L} / \mathrm{m}$ & $24.81(3.19)$ & $26.55(3.63)$ & $23.23(3.13)$ & $\mathbf{0 . 0 1 9}^{\#}$ \\
\hline Extracellular water, L & $19.66(3.54)$ & $20.87(3.82)$ & $18.27(3.02)$ & 0.125 \\
\hline \multicolumn{5}{|l|}{ Dietary intake } \\
\hline Calorie intake, Kcal/day & $2081.8(447.28)$ & 2376.63 (687.16) & 2043.11 (481.71) & 0.119 \\
\hline Protein intake, g/day & $79.84(19.92)$ & $84.66(22.31)$ & $76.09(23.85)$ & 0.486 \\
\hline Carbohydrates intake, \% & $51.05(5.08)$ & $49.56(8.92)$ & $47.44(3.81)$ & 0.484 \\
\hline Sugars, $\%$ & $17.9(4.22)$ & $19.12(4.75)$ & $17.56(6.48)$ & 0.524 \\
\hline Lipid intake, $\%$ & $31.3(5.31)$ & $32.54(7.42)$ & $31.67(4.92)$ & 0.776 \\
\hline SFA, $\%$ & $10.25(2.1)$ & $9.76(2.42)$ & $11(2.55)$ & 0.335 \\
\hline PUFA, $\%$ & $4.1(0.96)$ & $4.61(1.28)$ & $4.56(1.13)$ & 0.284 \\
\hline Water intake, mL/day & $1240(439.37)$ & $1219.51(498.1)$ & 1177.77 (263.52) & 0.945 \\
\hline Fibers intake, g/day & $28.25(8.91)$ & $32.41(8.02)$ & $25.31(9.64)$ & $\mathbf{0 . 0 3 9}^{\wedge}$ \\
\hline Calcium intake, mg/day & $879.45(241.89)$ & $970.71(326.64)$ & $906.11(300.5)$ & 0.517 \\
\hline Iron intake, intake, mg/day & $12.93(3.66)$ & $14.32(3.19)$ & $12.55(3.32)$ & 0.180 \\
\hline Zinc intake, mg/day & $11.32(2.81)$ & $12.27(2.94)$ & $10.87(3.27)$ & 0.298 \\
\hline Vitamin A intake, $\mu \mathrm{g} /$ day & $1172.85(377.43)$ & $1375.8(500.46)$ & $1146(507.07)$ & 0.185 \\
\hline Vitamin D intake, $\mu \mathrm{g} /$ day & $2.69(1.25)$ & $2.68(1.22)$ & $2.62(1.29)$ & 0.989 \\
\hline Vitamin E intake, mg/day & $12.67(2.95)$ & $16.54(5.6)$ & $12.07(3.82)$ & $0.004^{\mathrm{X}}$ \\
\hline Vitamin B12 intake, mg/day & $4.96(1.91)$ & $7.74(17.9)$ & $4.6(1.49)$ & 0.692 \\
\hline Vitamin $\mathrm{C}$ intake, $\mathrm{mg} /$ day & $156.5(74.34)$ & $192.68(62.21)$ & $147.11(72.7)$ & 0.060 \\
\hline Folate intake, $\mu \mathrm{g} /$ day & $347.59(104.41)$ & $416.24(112.02)$ & $321(112.48)$ & $0.017^{\wedge}$ \\
\hline Alcohol intake, g/day & $6.6(6.45)$ & $12.76(21.93)$ & $17.18(17.68)$ & 0.295 \\
\hline
\end{tabular}

Significant differences are highlighted in bold

Data are expressed in mean (standard deviation), unless otherwise specified

$H C$ healthy controls; PD Parkinson's disease; PSP Progressive supranuclear palsy; PUFA Poly-unsaturated fatty acids; SFA Saturated fatty acids *Difference between PSP and PD $p=0.001$; difference between PSP and HC $p=0.019$

${ }^{\S}$ Difference between PSP and PD $p<0.001$; difference between PSP and HC $p<0.001$

${ }^{\circ}$ Difference PSP and PD $p<0.001$; difference between PSP and HC $p=0.002$

${ }^{+}$Post hoc did not show significant differences between groups $(p>0.05)$

${ }^{\wedge}$ Difference between PD and HC $p=0.048$

\#Difference between PD and HC $p=0.033$

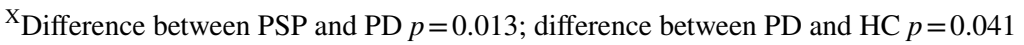


PD men and PSP women with PD women (Supplemental File 2).

Estimated rest energy expenditure was lower than measured rest energy expenditure. Mean (standard deviation, 95\% confidence intervals) difference between measured and estimated rest energy expenditure was 256.46 in the whole PSP cohort $(341.58,100.97-411.95)(p=0.003), 51.88$ in women $(305.52,-117.31-221.07)(p<0.001)$ and 194.66 in men $(340.42,77.72-311.6)(p<0.001)$.

In general, the Harris-Benedict formula tended to underestimate rest energy expenditure in PSP by $15.1 \%$. The Lin's concordance correlation coefficient (95\% confidence intervals) was $0.471(0.164-0.695)$ showing only moderate agreement between measured and estimated rest energy expenditure. The proportion of accurate prediction $( \pm 10 \%$ difference between measured and estimated rest energy expenditure) in the whole population was $42.8 \%$. In general, the equation was likely to underestimate rest energy expenditure $(<10 \%$ in $47.6 \%$ of cases $;>10 \%$ in $9.5 \%$ of cases $)$.

\section{Body composition}

PSP tended to present lower Free Fat Mass and Free Fat Mass index compared to PD without reaching the threshold for a significant difference $(p>0.05)$. When dividing the patients by sex, such trend was confirmed in men $(p>0.05)$ (Supplemental File 2). Pathologic skeletal muscle mass index had a low prevalence in both PSP and PD cohorts (9.5\% and 5\%, respectively). No significant differences in pathological skeletal muscle mass were shown when dividing the patients by sex (Supplemental File 2).

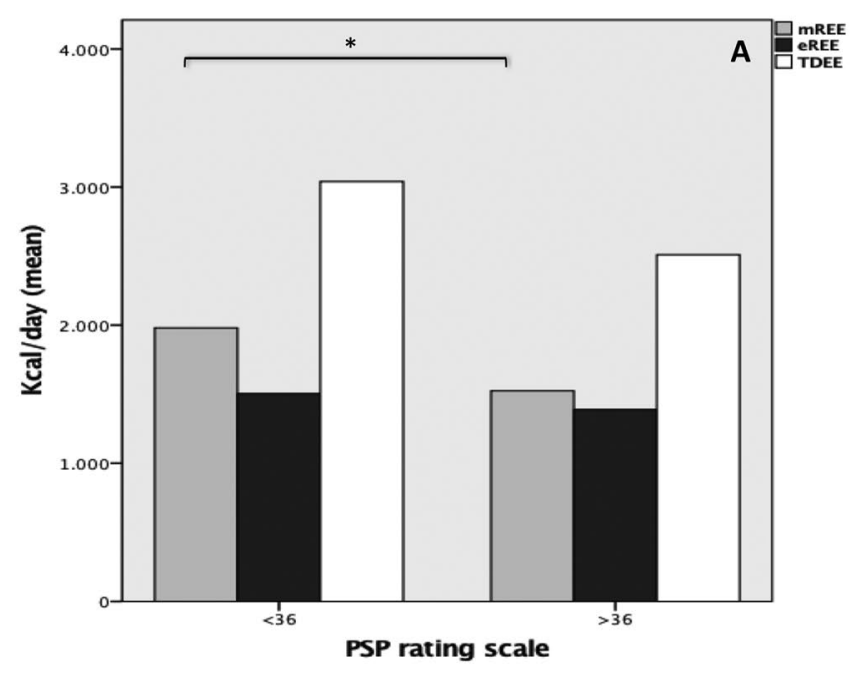

Fig. 1 Differences in energy expenditure (A) and body composition (B) according to disease severity in PSP. *: $p<0.05$; $e$ REE estimated Rest Energy Expenditure; FFM Free Fat Mass; $m R E E$ measured Rest

\section{Dietary intake}

PSP patients had lower vitamin E intake compared to PD $(p<0.05)$. No other significant differences in dietary intake for PSP were shown.

\section{Differences according to pre-specified categories in the PSP cohort}

Supplemental File 2 provides details for differences according to pre-specified categories in the PSP cohort.

As for sex differences, in line with data available in healthy controls, rest energy expenditure, Free Fat Mass and skeletal muscle mass index, Total Body Water and Extracellular water were higher in men, while Fat Mass index was higher in women.

No significant differences were shown according to diseases phenotypes.

As for disease severity, patients with higher PSP-rs showed lower rest energy expenditure, Free Fat Mass, Free Fat Mass index, skeletal muscle mass index, Total Body Water and Total Body Water index compared to patients with lower PSP-rs. Furthermore, patients with a more severe form of disease showed a tendency toward lower total daily energy expenditure (Fig. 1A, B). No significant differences were present for either anthropometric data or dietary habits.

No significant differences were shown in patients with clinically relevant dysphagia except for a lower intake of saturated fatty acid and a tendency for lower water intake compared to patients without clinically relevant dysphagia.

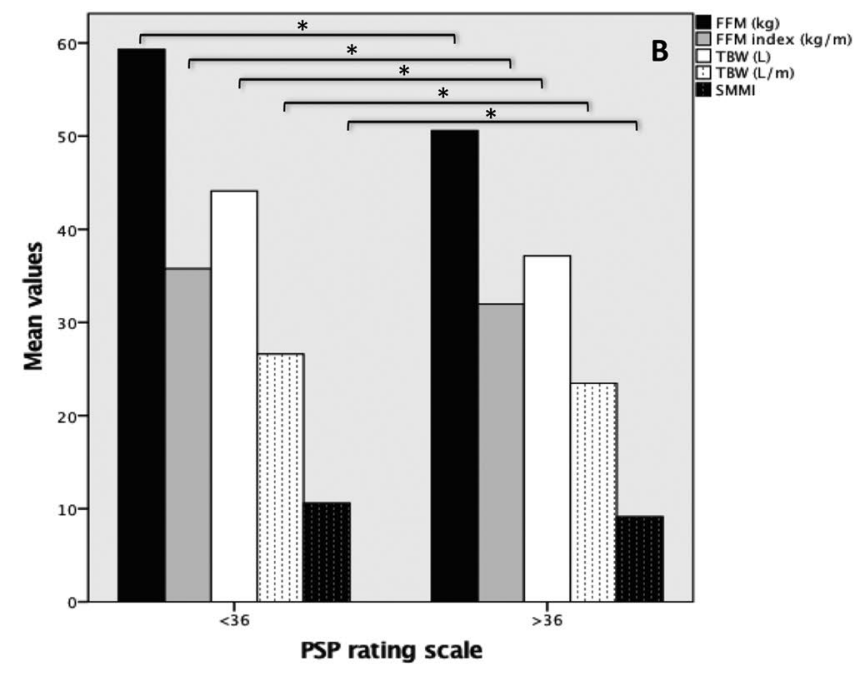

Energy Expenditure; SMMI Skeletal Muscle Mass Index; TBW Total Body Water 


\section{Discussion}

Using an extensive set of instrumental assessments and validated questionnaires, we investigated energy expenditure, body composition and dietary intake in PSP at early stages in comparison with age-, sex- and BMI-matched PD patients and HC.

PSP disclosed similar measured and estimated rest energy expenditure, but lower total daily energy expenditure compared to PD and HC. The significant reduction in total daily energy expenditure in PSP was likely due to the significant contraction of physical activity demonstrated by the reduction of both physical activity level and physical activity intensity levels. Indeed, such reduced mobility is likely due to the high motor disability and risk of falls experienced by PSP patients since the earliest stages of disease [22]. In keeping with this speculation, motor disability of the PSP cohort is supported by the mean (standard deviation) scores with the Fall, Gait and Postural Instability items of the PSP-rs [2.29 (1.19), 1.9 (0.89), 2.24 (1.14), respectively)]. Differently from PSP, bvFTD and ALS have been associated with a hypermetabolic status with increased resting energy expenditure [5, 23]. Yet, although PSP belongs to the FTLD spectrum as bvFTD and ALS, our data would suggest that PSP at early stages is not associated with major changes in rest energy expenditure as compared with PD and HC.

Our data also demonstrated that the Harris-Benedict equation does not provide a reliable estimation of rest energy expenditure in PSP. Although extensively validated in healthy controls, in the present study, the Harris-Benedict equation underestimated measured energy expenditure also in PD and healthy controls. Limited accuracy of such equation in PSP is also supported by the scarce agreement between measured and estimated rest energy expenditure shown by the Lin's concordance correlation coefficient. As a consequence, the Harris-Benedict equation should not be considered as a valid proxy of indirect calorimeter assessment in PSP in routine practice. Reliability of the other available equations should be tested in PSP to verify their validity in the estimation of measured rest energy expenditure. Alternatively, new prediction equations should be designed by future studies taking into account the predictors of estimation bias [3].

As for body composition, we showed PSP did not have major significant differences compared with PD and HC. In keeping with the discrepancy observed for energy expenditure, also body composition changes in PSP differ from those observed in bvFTD [6]. Ahmed et al. reported an increase in both total lean and fat mass in bvFTD compared to both Alzheimer's disease and HC [6]. Differently from Ahmed et al., we only observed a tendency toward a reduction of Free Fat Mass and skeletal muscle mass index in PSP compared with PD. On the other hand, we confirmed the low prevalence of pathologic skeletal muscle mass index in PD and parkinsonism [2].

All our results on basal metabolism and body composition were confirmed when dividing PSP and PD cohorts by sex. Takamatsu et al. found lower basal metabolism only in women with PSP compared to age-matched healthy women [24]. Such discrepancy with our data may be explained by few methodological differences. First, Takamatsu et al. performed a retrospective estimation of body metabolism with a body composition analyzer while we measured it with a calorimeter in a prospective study. Furthermore, Takamatsu et al. also included in their study inpatients performing rehabilitation, while our cohort only included outpatients. Finally, due to the small number of healthy controls in our study, we run comparisons by sex between PSP and PD only.

As for dietary intake and in keeping with similar anthropometric data, we failed to find major differences in caloric intake, macronutrients and micronutrients assumptions in PSP compared to both PD an HC, except for a lower intake of vitamin E compared to PD. Such findings may suggest dietary intake does not have a major role in the differences observed for energy expenditure and body composition.

As for the evaluation of energy expenditure, body composition and dietary intake according to pre-specified PSP subgroups, we confirmed men with PSP have higher rest energy expenditure and Fat-Free Mass and lower Fat Mass compared to women with PSP in line with sex differences in the general population.

When dividing the PSP cohort according with disease phenotypes, we failed to show major differences. However, the PSP cohort was mainly represented by PSP-RS (80.9\%), thus, our study lacks of the necessary power to demonstrate any differences at the sub-group level $[10,11]$.

Interestingly and differently from bvFTD [5], we showed that disease severity, as measured with the PSP rating scale, was associated with lower measured but not estimated rest energy expenditure, further supporting the scarce agreement between these two measures. As a significant contraction in physical activity was lacking, such reduction in rest energy expenditure in PSP patients with a more severe form of disease was likely responsible for a trend toward significance for lower total daily energy expenditure. As for body composition, differently from bvFTD and in spite of similar anthropometric measures (i.e., weight, BMI, waist circumference) [6], greater disease severity was associated with lower Fat-Free Mass and Fat-Free Mass index, skeletal muscle mass index, Total Body Water, and Total Body Water index. Based on available literature on healthy individuals [25], we cannot exclude reduced muscle mass may be associated with a reduction in rest energy expenditure in patients with greater severity of disease. Given the lack of difference 
in protein intake, we hypothesize the tendency toward a reduced mobility might play a role in body composition changes affecting the PSP patients with disease progression.

Indeed, skeletal muscle mass index reduction has a key role in determining sarcopenia, which in turn is linked to loss of muscle strength (dynapenia), further contributing to reduction in mobility and increasing motor difficulties in elderly patients $[2,26]$. Recent randomized clinical trials have shown that in sarcopenic older adults oral nutritional support with muscle-targeted whey protein-based formula enriched with essential amino acids may improve muscle mass, strength and physical function, especially in combination with physical therapy [27-29]. Recently, Barichella et al. showed the efficacy of a whey protein-based nutritional formula associated with physical rehabilitation in preserving muscle mass and improving motor performances in patients with parkinsonism [30]. Although few PSP patients were included in this study (8-9\%) [30], strong evidence for efficacy of muscle-targeted nutritional support on disease severity in PSP is not available yet. Indeed, the data of the present study would support the rationale for the design of such a clinical trial in PSP patients at early stages.

Given such changes in energy expenditure and body composition with disease severity can only be detected with objective instrumental assessments (i.e., indirect calorimeter and bio-impedance analysis) and are not associated to change in anthropometric measures or dietary intake, they are at high risk of being unrecognized by clinicians and, thus, undertreated.

Finally, we failed to detect major differences in any of the administered assessments in relation with the presence of clinically significant dysphagia. Since we recruited a population of early PSP, we speculate dysphagia was not severe enough yet to determine significant changes in patients' metabolism.

A complementary or alternative explanation for the change in energy expenditure and body composition observed in PSP patients with greater disease severity is autonomic dysfunction. The presence of autonomic dysfunction in PSP is increasingly recognized with a length-dependent loss of sensory and autonomic nerve fibers associated with functional impairment related to autonomic dysfunction paralleling disease severity [31]. Such hypothesis requires further studies as we did not evaluate autonomic function in the present study.

Our study has limitations. We are aware a limited number of $\mathrm{HC}$ was included in the present study. The main reason for low enrollment of HC was COVID-19 pandemic which limited the access and the time spent into the health care facility for spouses and caregivers. Future studies should enroll a larger healthy control group with no direct link to the patient population. Also, we cannot exclude the COVID-19 pandemic may have had an impact on measurements performed in the present study. Lockdown restrictions may have contributed to decreased daily energy expenditure and physical activity levels, particularly in the PSP group. However, we failed to find significant differences in the outcome measures between patients enrolled before and after the COVID-19 pandemic (data not shown). Furthermore, we acknowledge a possible assessment bias, with either under- or over-reporting, associated with the method used in the ascertainment of dietary habits as well as with physical activity level [1]. Notwithstanding, all questionnaires and equations used in the present work have been validated and extensively applied to the general population and to PD patients in Italy and worldwide [1-3]. Furthermore, we decided to use the cutoff for sarcopenia recommended by the European consensus and used in previous works including Italian parkinsonian subjects $[2,26,30]$. Notwithstanding, we are aware the cutoff for sarcopenia relies on the measurement method and on the population involved. Also, all the patients included in the present study were recruited in a single center in the South of Italy. Thus, we recognize dietary habits and anthropometric data may not necessarily reflect those from the general population of PSP patients. Another drawback is the cross-sectional design of our study. Further longitudinal studies are required to confirm the relationship between changes in energy expenditure and body composition and disease progression and the effect on overall survival in PSP. Finally, our study only provides a first extensive description of energy expenditure, body composition and dietary habits in PSP. Future studies will also apply neuroimaging techniques to explore the pathophysiological correlates of such metabolic changes in PSP at early stages.

In conclusion, we provide data on an extensive set of assessments evaluating energy expenditure, body composition and dietary intake in PSP at early stages. We showed lower total daily energy expenditure possibly linked to reduced mobility in PSP compared with age-, sex-, BMImatched PD and HC associated with trend for a reduction in lean mass. Furthermore, we demonstrated the Harris-Benedict equation, a validated method to estimate rest energy expenditure in healthy controls, does not provide a reliable estimation of measured rest energy expenditure in PSP. Finally, we showed disease severity in PSP is linked with a reduction in rest energy expenditure possibly due to the significant reduction in lean and muscle mass. If confirmed by larger and longitudinal studies, such data may pave the way to future clinical trials evaluating the efficacy of muscle-targeted nutritional support with or without physical therapy in preserving muscle mass and improving measures of disease severity in PSP at early stages.

Supplementary Information The online version contains supplementary material available at https://doi.org/10.1007/s00415-021-10846-6. 
Acknowledgements We are grateful to the patients who participated in the study.

Author contributions MP: Research project: conception, organization; Statistical analysis: design, execution; Manuscript: writing of the first draft. MFT, MCR, FA, MS, ADS: Research project: execution; Manuscript: review and critique. RE, MTP, PB: Statistical analysis: review and critique; Manuscript: review and critique.

Funding The present was supported with FARB2020, University of Salerno, Italy.

\section{Declarations}

Conflict of interest The authors declare that they have no conflict of interests. Statistical analysis was conducted by Marina Picillo, MD, PhD. Disclosures from the past two years: Dr Marina Picillo is supported by the Michael J Fox Foundation for Parkinson's research; Prof Paolo Barone received consultancies as a member of the advisory board for Zambon, Lundbeck, UCB, Chiesi, Abbvie and Acorda; Dr Roberto Erro received consultancies from Zambon and honoraria from TEVA; the other authors report no financial diclosures.

Ethical approval The study was approved by the institutional Ethics Committee (N. 101, 19 October 2017) and was carried out in accordance with the principles laid down in the 1964 Declaration of Helsinki and later amendments. Subjects were recruited upon signature of the written consent form.

\section{References}

1. Barichella M, Cereda E, Cassani E, Pinelli G, Iorio L, Ferri V, Privitera G, Pasqua M, Valentino A, Monajemi F, Caronni S, Lignola C, Pusani C, Bolliri C, Faierman SA, Lubisco A, Frazzitta G, Petroni ML, Pezzoli G (2017) Dietary habits and neurological features of Parkinson's disease patients: implications for practice. Clin Nutr 36(4):1054-1061

2. Barichella M, Pinelli G, Iorio L, Cassani E, Valentino A, Pusani C, Ferri V, Bolliri C, Pasqua M, Pezzoli G, Frazzitta G, Cereda E (2016) Sarcopenia and dynapenia in patients with Parkinson's disease. J Am Med Dir Assoc 17(7):640-646

3. Barichella M, Cereda E, Faierman SA, Piuri G, Bolliri C, Ferri V, Cassani E, Vaccarella E, Donnarumma OV, Pinelli G, Caronni S, Pusani C, Pezzoli G (2020) Resting energy expenditure in Parkinson's disease patients under dopaminergic treatment. Nutr Neurosci 7:1-10

4. Ahmed RM, Irish M, Piguet O, Halliday GM, Ittner LM, Farooqi S, Hodges JR, Kiernan MC (2016) Amyotrophic lateral sclerosis and frontotemporal dementia: distinct and overlapping changes in eating behaviour and metabolism. Lancet Neurol 15(3):332-342

5. Ahmed RM, Landin-Romero R, Collet TH, van der Klaauw AA, Devenney E, Henning E, Kiernan MC, Piguet O, Farooqi IS, Hodges JR (2017) Energy expenditure in frontotemporal dementia: a behavioural and imaging study. Brain 140:171-183

6. Ahmed RM, Landin-Romero R, Liang CT, Keogh JM, Henning E, Strikwerda-Brown C, Devenney EM, Hodges JR, Kiernan MC, Farooqi IS, Piguet O (2019) Neural networks associated with body composition in frontotemporal dementia. Ann Clin Transl Neurol 6(9):1707-1717

7. Tsuge A, Kaneko S, Wate R, Oki M, Nagashima M, Asayama S, Nakamura M, Fujita K, Saito A, Takenouchi N, Kusaka H (2016)
Weight loss in the early stage of progressive supranuclear palsy. Brain Behav 7(2):e00616

8. Martinelli P, Scaglione C, Lodi R, Iotti S, Barbiroli B (2000) Deficit of brain and skeletal muscle bioenergetics in progressive supranuclear palsy shown in vivo by phosphorus magnetic resonance spectroscopy. Mov disord 15(5):889-893

9. Hoglinger GH, Respondek G, Stamelou M, Kurz C, Josephs KA, Lang AE, Mollenhauer B, Müller U, Nilsson C, Whitwell JL, Arzberger T, Englund E, Gelpi E, Giese A, Irwin DJ, Meissner WG, Pantelyat A, Rajput A, van Swieten JC, Troakes C, Antonini A, Bhatia KP, Bordelon Y, Compta Y, Corvol JC, Colosimo C, Dickson DW, Dodel R, Ferguson L, Grossman M, Kassubek J, Krismer F, Levin J, Lorenzl S, Morris HR, Nestor P, Oertel WH, Poewe W, Rabinovici G, Rowe JB, Schellenberg GD, Seppi K, van Eimeren T, Wenning GK, Boxer AL, Golbe LI, Litvan I, Movement Disorder Society-endorsed PSP Study Group (2017) Clinical diagnosis of progressive supranuclear palsy: the movement disorder society criteria. Mov Disord 32:853-864

10. Picillo M, Erro R, Cuoco S, Tepedino MF, Manara R, Pellecchia MT, Barone P, PSP Salerno Study Group (2018) MDS PSP criteria in real-life clinical setting: motor and cognitive characterization of subtypes. Mov Disord 33(8):1361-1365

11. Picillo M, Cuoco S, Tepedino MF, Cappiello A, Volpe G, Erro R, Santangelo G, Pellecchia MT, Barone P, PSP Salerno study group (2019) Motor, cognitive and behavioral differences in MDS PSP phenotypes. J Neurol 266(7):1727-1735

12. Picillo M, Tepedino MF, Abate F, Erro R, Ponticorvo S, Tartaglione S, Volpe G, Frosini D, Cecchi P, Cosottini M, Ceravolo R, Esposito F, Pellecchia MT, Barone P, Manara R (2020) Midbrain MRI assessments in progressive supranuclear palsy subtypes. J Neurol Neurosurg Psychiatry 91(1):98-103

13. Grimm M-J, Respondek G, Stamelou M, Arzberger T, Ferguson L, Gelpi E, Giese A, Grossman M, Irwin DJ, Pantelyat A, Rajput A, Roeber S, van Swieten JC, Troakes C, Antonini A, Bhatia KP, Colosimo C, van Eimeren T, Kassubek J, Levin J, Meissner WG, Nilsson C, Oertel WH, Piot I, Poewe W, Wenning GK, Boxer A, Golbe LI, Josephs KA, Litvan I, Morris HR, Whitwell JL, Compta Y, Corvol JC, Lang AE, Rowe JB, Höglinger GU, Movement Disorder Society-endorsed PSP Study Group (2019) How to apply the movement disorder Society criteria for diagnosis of progressive supranuclear palsy. Mov Disord 34:1228-1232

14. Postuma RB, Berg D, Stern M, Poewe W, Olanow CW, Oertel W, Obeso J, Marek K, Litvan I, Lang AE, Halliday G, Goetz CG, Gasser T, Dubois B, Chan P, Bloem BR, Adler CH, Deuschl G (2015) MDS clinical diagnostic criteria for Parkinson's disease. Mov Disord 30:1591-1601

15. World Health Organization (1995) Physical status: the use and interpretation of anthropometry. Report of a WHO expert committee. World Health Organ Tech Rep Ser 854:1-452

16. Janssen I, Baumgartner RN, Ross R, Rosenberg IH, Roubenoff R (2004) Skeletal muscle cutpoints associated with elevated physical disability risk in older men and women. Am J Epidemiol 159:413-421

17. Chien MY, Huang TY, Wu YT (2008) Prevalence of sarcopenia estimated using a bioelectrical impedance analysis prediction equation in community-dwelling elderly people in Taiwan. J Am Geriatr Soc 56:1710-1715

18. Antonini A, Abbruzzese G, Ferini-Strambi L, Tilley B, Huang J, Stebbins GT, Goetz CG, Barone P, MDS-UPDRS Italian Validation Study Group, Bandettini di Poggio M, Fabbrini G, Di Stasio F, Tinazzi M, Bovi T, Ramat S, Meoni S, Pezzoli G, Canesi M, Martinelli P, Maria Scaglione CL, Rossi A, Tambasco N, Santangelo G, Picillo M, Morgante L, Morgante F, Quatrale R, Sensi M, Pilleri M, Biundo R, Nordera G, Caria A, Pacchetti C, Zangaglia R, Lopiano L, Zibetti M, Zappia M, Nicoletti A, Quattrone A, Salsone M, Cossu G, Murgia D, Albanese A, Del Sorbo F 
(2013) Validation of the Italian version of the Movement Disorder Society-Unified Parkinson's Disease Rating Scale. Neurol Sci 34(5):683-687

19. Golbe LI, Ohman-Strickland PA (2007) A clinical rating scale for progressive supranuclear palsy. Brain 130(Pt 6):1552-1565

20. Bland JM, Altman DG (1995) Comparing methods of measurement: why plotting difference against standard method is misleading. Lancet 346(8982):1085-1087

21. Lin LI (1989) A concordance correlation coefficient to evaluate reproducibility. Biometrics 45(1):255-268

22. Srulijes K, Klenk J, Schwenk M, Schatton C, Schwickert L, Teubner-Liepert K, Meyer M, Srijana KC, Maetzler W, Becker C, Synofzik M (2019) Fall risk in relation to individual physical activity exposire in patients with different neurodegenerative diseases: a pilot study. Cerebellum 18(3):340-348

23. Bouteloup C, Desport JC, Clavelou P, Guy N, DerumeauxBurel H, Ferrier A, Couratier P (2009) Hypermetabolism in ALS patients: an early and persistent phenomenon. J Neurol 256:1236-1242

24. Takamatsu Y, Aiba I (2021) Skeletal muscle loss and body composition in progressive supranuclear palsy: a retrospective crosssectional study. PLoS ONE 16(6):e0253079

25. Pontzer H, Yamada Y, Sagayama H, Ainslie PN, Andersen LF, Anderson LJ et al (2021) Daily energy expenditure through the human life course. Science 373(6556):808-812

26. Cruz-Jentoft AJ, Landi F, Schneider SM, Zuniga C, Arai H, Boirie Y, Chen L, Fielding RA, Martin FC, Michel J, Sieber C, Stout JR, Studenski SA, Vellas B, Woo J, Zamboni M, Cederholm T (2014) Prevalence of and interventions for sarcopenia in ageing adults: a systematic review. Report of the International Sarcopenia Initiative (EWGSOP and IWGS). Age Ageing 43:748-759
27. Bauer JM, Verlaan S, Bautmans I, Brandt K, Donini LM, Maggio M, McMurdo ME, Mets T, Seal C, Wijers SL, Ceda GP, De Vito G, Donders G, Drey M, Greig C, Holmbäck U, Narici M, McPhee J, Poggiogalle E, Power D, Scafoglieri A, Schultz R, Sieber CC, Cederholm T (2015) Effects of a vitamin D and leucine-enriched whey protein nutritional supplement on measures of sarcopenia in older adults, the PROVIDE study: a randomized, double-blind, placebo-controlled trial. J Am Med Dir Assoc 16:740-747

28. Rondanelli M, Klersy C, Terracol G, Talluri J, Maugeri R, Guido D, Falliva MA, Solerte BS, Fioravanti M, Lukaski H, Perna S (2016) Whey protein, amino acids, and vitamin supplementation with physical activity increases fat-free mass and strength, functionality, and quality of life and decreases inflammation in sarcopenic elderly. Am J Clin Nutr 103:830-840

29. Dimori S, Leoni G, Fior L, Gasparotto F (2018) Clinical nutrition and physical rehabilitation in a long-term care setting: preliminary observations in sarcopenic older patients. Aging Clin Exp Res 30:951-958

30. Barichella M, Cereda E, Pinelli G, Iorio L, Caroli D, Masiero I, Ferri V, Cassani E, Bolliri C, Caronni S, Maggio M, Ortelli P, Ferrazzoli D, Maras A, Riboldazzi G, Frazzitta G, Pezzoli G (2019) Muscle-targeted nutritional support for rehabilitation in patients with parkinsonian syndrome. Neurology 93(5):485-496

31. Dubbioso R, Provitera V, Vitale F, Stancanelli A, Borreca I, Caporaso G, De Michele G, De Rosa A, Picillo M, Barone P, Iodice R, Manganelli F, De Michele G, Santoro L, Nolano M (2021) Cutaneous sensory and autonomic denervation in progressive supranuclear palsy. Neuropathol Appl Neurobiol 47(5):653-663 